\title{
Pensar y hacer futuro en educación. Hacia un proyecto curricular y pedagógico centrado en la adquisición y desarrollo de capacidades ${ }^{\star}$
}

\author{
Horacio Ademar Ferreyra ${ }^{* *}$, Gabriela Peretti*** Silvia Vidales ${ }^{* * * *}$
}

\begin{abstract}
Resumen
Recibido: 25 de febrero de 2011 Revisado: 23 de marzo de 2011 Aprobado: 1 de abril de 2011

El aprendizaje centrado en el desarrollo de capacidades es susceptible de nuevas reflexiones si se lo sitúa en las coordenadas del pensar la educación para construir futuro. La discusión cobra sentido en un escenario signado por la importancia de la significación y relevancia del conocimiento, y la necesidad de asegurar el principio de igualdad de oportunidades en función de las potencialidades y estilos de aprendizaje de los estudiantes.
\end{abstract}

A la luz de los propósitos de democratizar el acceso y apropiación del conocimiento, construir ciudadanía y humanizar, se sostiene

* El presente artículo recupera, para enriquecerlas e interpelarlas, algunas consideraciones ya desarrolladas en la: a) conferencia magistral Desarrollo de competencias educativas prioritarias, pronunciada por el doctor Horacio Ferreyra en la Pontificia Universidad Católica, sede Paraná, Argentina, 2003; b) ponencia Desarrollo de capacidades fundamentales realizada en el Congreso Metas 2021-OEl, Buenos Aires, Argentina, 2010, por Horacio Ferreyra y Gabriela Peretti; c) Ferreyra, H., Peretti, G., Vidales, S. y Alegre (2010). Desarrollo de capacidades fundamentales: aprendizaje relevante y educación para toda la vida. En Ferreyra, H. (Ed.). Actualidad y campo pedagógico. Textos con el pretexto de habilitar el debate (en prensa). Bogotá: Universidad Santo Tomás.

* Doctor en Educación de la Universidad Católica de Córdoba, Argentina, Posdoctorado en Ciencias Sociales en el Centro de Estudios Avanzandos de la Universidad Nacional de Córdoba, Argentina y Posdoctorado en Ciencias Sociales de la Universidad Autónoma Metropolitana, México. Docente e investigador en la Facultad de Educación de la Universidad Católica de Córdoba, Argentina y Facultad de Educación de la Universidad Santo Tomás, Colombia, y es subsecretario de Promoción de Igualdad y Calidad Educativa en el Ministerio de Educación de la Provincia de Córdoba, Argentina. Correo electrónico: hferreyra@coopmorteros.com.ar

*** Profesora en Psicología y en Ciencias de la Educación y candidata a magíster en la Maestría en Gestión Educacional en la Universidad del Mar (Chile). Actualmente se desempeña como responsable del área de Gestión Curricular del ME. Cba. Fue miembro del equipo técnico DEMyS (1999 - 2001), coordinadora pedagógica del PNBE (2002 - 2004) y evaluadora del proyecto de la Subunidad de ejecución y gestión del sistema educativo (2005 - 2007) en el ME. Cba. Correo electrónico: sajucateumaco@yahoo.com.ar

**** Profesora y licenciada en Letras Modernas de la Universidad Nacional de Córdoba. Estudios de posgrado (especialidad) en Enseñanza en Educación Superior. Docente de Comunicación Oral y Escrita en diferentes carreras de la Universidad Empresarial Siglo 21 y Universidad Blas Pascal, Córdoba, Argentina. Profesora en Institutos Superiores de Enseñanza en la formación de docentes de educación primaria y secundaria en Lengua y Literatura. Se ha desempeñado como capacitadora de la Red Federal de Formación Docente Continua y como asesora de instituciones educativas en la construcción del Proyecto Curricular Institucional y en el diseño e implementación de proyectos específicos de enseñanza y evaluación de la Lengua y la Literatura. Correo electrónico: silvidales@gmail.com 
una perspectiva que concibe a las capacidades asociadas a procesos tanto cognitivos como socioafectivos que garantizan la formación integral. A partir de ello, se propone la construcción de proyectos curriculares y pedagógicos enmarcados en políticas educativas inclusivas, preocupadas por la calidad, relevancia y pertinencia de los saberes y orientadas al crecimiento y mejora de los sujetos y las comunidades.

\section{Palabras clave}

Civilización sociocognitiva, proyecto educativo, capacidades fundamentales, educación permanente, ciudadanía. 


\title{
Thinking and Making Future Education. Towards a Curricular and Pedagogical Project Focused on the Acquisition and Development Capabilities
}

\author{
Horacio Ademar Ferreyra, Gabriela Peretti, Silvia Vidales
}

Recibido: 25 de febrero de 2011 Revisado: 23 de marzo de 2011 Aprobado: 1 de abril de 2011

\section{Abstract}

The learning that is centered on capacities is subject to new thought when set in the coordinates of "thinking in education" to build a future. This discussion acquires significance in a scenario signed by the importance of significance and the relevance of knowledge and the need to guarantee the principle of equal opportunities according to the potentialities and learning styles of the students.

In the light of the aims to democratize the access to and the acquisition of knowledge, to build citizenship and to humanize, a perspective, which conceives the capacities related to cognitive as well as socio-affective processes that guarantee an integral training, is held. From there on, we propose the construction of curricular and pedagogical projects framed in inclusive and educational policies caring for the quality, relevance and pertinence of the knowledges and oriented to the development and improvement of the students and their communities.

\section{KeYwORDS}

Sociocognitive civilization, educational project, fundamental capacity, permanent education, citizenship. 


\section{INTRODUCCIÓN}

Pensar la educación es, primordialmente, pensar el futuro y en el futuro. Esta afirmación, para nada original en tanto conocido leiv motiv de numerosos discursos en torno al campo educativo, puede ser objeto de nuevas construcciones de sentido si, en vez de centrar inmediatamente la atención en la palabra futuro, nos detenemos, primero, en pensar. Y ésta es, precisamente, la impronta que pretendemos conferir a este artículo que aborda una temática que tampoco es novedosa -el desarrollo de capacidades-, pero que entendemos es todavía susceptible de nuevas reflexiones si la situamos en las coordenadas del pensar la educación para construir futuro.

Pensar (del latín pensare) admite -según lo consigna el Diccionario de la Real Academia de la Lengua Española- diversas acepciones, todas ellas productivas para las intencionalidades reflexivas de este artículo:

\section{Imaginar, considerar o discurrir.}

2. Reflexionar, examinar con cuidado algo para formar dictamen.

3. Intentar o formar ánimo de hacer algo.

Entonces, el propósito es abordar algunos aportes en torno a la pertinencia y la posibilidad de construir un Proyecto Educativo orientado al desarrollo de capacidades que suponga un camino posible para (re)pensar la educación desde las dimensiones del discurrir imaginativo, de la reflexión para la toma de decisiones y de la iniciativa para hacer un futuro mejor.

\section{COMPETENCIAS Y CAPACIDADES, DEBATES Y PRECISIONES EN UN NUEVO ESCENARIO EDUCATIVO}

Concentrarnos sólo en los debates en torno a los conceptos de capacidad y de competencia, muchas veces conduce a soslayar una lectura en clave histórica que permitiría apreciar que la controversia entre estos conceptos sólo ha sido posible a partir de una circunstancia particularmente feliz en el ámbito de la educación: la superación de la primacía de las conductas, evidencias "que debían observarse, demostrarse, y evaluarse con registros 'objetivos', tras situaciones de enseñanza magistral dirigidas al 'alumno-medio', desde el apremio del 'programa', y procesos de aprendizaje resueltamente memorísticos" (Montero, 2008, p. 35). Sobre esta superación se construye un escenario en el que cobran relevancia las teorías que acentúan la significación y relevancia del conocimiento que se construye en un proceso personal de aprendizaje, mediado por la intervención docente y las múltiples interacciones (de los sujetos entre sí y con los contextos) que supone el hecho educativo. $Y$ éste es también el escenario en el que comienza a construir su espacio, el principio de igualdad de oportunidades y a cobrar sentido la noción de trayecto educativo, entendido como el recorrido que todos y cada uno de los sujetos -en función de sus potencialidades, estilos y ritmos de aprendizaje- puede realizar cuando se le habilitan oportunidades de alcanzar -en contexto- las metas previstas para todos.

Por otra parte, ocuparnos de discurrir en torno al sentido y los alcances de los términos "capacidad" y "competencia" con 
referencia a su incidencia en el campo educativo, en general; y pedagógico, en particular, exige también una mirada hacia los nuevos órdenes y contextos. La caracterización de la sociedad actual como civilización sociocognitiva supone fenómenos que atraviesan lo social, lo político, lo ético, lo económico, lo cultural, con diversidad de efectos y consecuencias en todos los campos, entre ellos el educativo. Surgen así nuevas demandas que exigen una redefinición de las concepciones de enseñanza, de aprendizaje, de contenido escolar, de sus interrelaciones; demandas que ponen en evidencia la necesidad de diversas renovaciones en los modelos didácticos.

Con el declarado propósito de "superar este debate de fuertes características ideológicas y de evitar distorsiones a partir de los múltiples discursos en torno a la cuestión de las competencias y las capacidades..." (Montero, 2008, p. 51), un documento producido por el Ministerio de Educación de Argentina, en concurrencia con UNICEF, OEI y la Asociación Civil Educación para todos, publicado en 2010, avanza hacia "algunas propuestas técnico-metodológicas con el fin de enmarcar los conjuntos de documentos y prácticas curriculares que se vienen desarrollando con referencia a estos términos" (Montero, 2008, p. 51). Se analizan, así, tresrelaciones que muestran los distintos sentidos que suelen darse a estos términos en ámbitos educativos:

a) Las competencias consideradas como equivalentes a capacidades.

b) Las competencias como resultado de la concurrencia de un conjunto de capacidades concurrentes. c) Las competencias como punto de llegada de un trayecto de desarrollo gradual de capacidades.

Desde la perspectiva que orienta este artículo, "capacidad"1 implica una cualidad o conjunto de cualidades de las personas, cuyo desarrollo y adquisición les permite enfrentar la realidad en condiciones más favorables. En tanto potencialidades inherentes a los sujetos y que estos procuran desarrollar a lo largo de toda su vida, suelen clasificarse como macrohabilidades o habilidades generales, talentos o condiciones de las personas, que les permiten tener un mejor desempeño o actuación en la vida cotidiana.

En esta perspectiva trasciende la concepción de las capacidades como estrategias cognitivas, pues las entiende asociadas a procesos cognitivos y socio-afectivos, que garantizan la formación integral de la persona; se manifiestan a través de un contenido o conjunto de ellos y constituyen, en este sentido, una base desde la cual se siguen procesando, incorporando y produciendo nuevos conocimientos.

Se pueden alcanzar diferentes grados de desarrollo de una capacidad y ésta se va perfeccionando con la práctica. En este sentido, una capacidad es un aprendizaje permanente que, según su nivel de evolución y perfeccionamiento, supone el manejo adecuado de determinadas destrezas y habilidades.

1 A diferencia de la capacidad, la "competencia" es inherente al mundo social: es estructurada y demandada desde fuera del sujeto, por el medio, por terceros y esencialmente por el mercado o "los mercados" (de consumo, del trabajo, profesional). Por lo tanto, puede ir cambiando de contenido conforme van desarrollándose los procesos histórico-sociales de una determinada comunidad organizada e implica poner en práctica técnicas y conocimientos relativos al "saber hacer", ciertas predisposiciones definidas desde fuera de las instituciones educativas y a las cuales, supuestamente, ellas mismas tendrían que adecuarse para reformular sus prácticas curriculares (para ampliar, véase Ministerio de Educación, Ciencia y Tecnología, 2011 y Ministerio de Educación, 2010, Argentina). 
Uno de los rasgos característicos de las capacidades es su complejidad, en cuanto entrañan una serie de operaciones o procesos interiores de distintos grados de interrelación mutua. Por otra parte, su posesión habilita a las personas a usarlas en variadas situaciones, es decir, no se ajustan a un patrón único de actuación, sino que posibilitan un manejo contextualizado que depende de la persona que las utiliza.

Existe también discusión acerca de si corresponde hablar de construcción o de desarrollo de capacidades. Al respecto, consideramos que el segundo término -que implica un proceso orgánico de crecimiento y progreso- es más apropiado que el de "construcción", el cual supone actividades que son planificadas y ejecutadas cuidadosamente de acuerdo con un proyecto minucioso y acabado, dirigido exclusivamente desde "fuera" de la persona del estudiante. Así, el desarrollo y adquisición de capacidades no puede "realizarse" sólo desde afuera: un docente puede informar y proporcionar ayudas al estudiante con la intención de promover o estimular el desarrollo y adquisición de capacidades, pero los mejores resultados implican la definición de objetivos y estrategias basados en intereses mutuos y en colaboración, así como una ejecución de planes flexibles, que permitan modificar lo planificado, a medida que las condiciones cambian y se avanza en el desarrollo.

\section{DesarRollar CAPACIDADES PARA DEMOCRATIZAR EL ACCESO Y APROPIACIÓN DEL CONOCIMIENTO, CONSTRUIR CIUDADANÍA Y HUMANIZAR}

En reiteradas ocasiones se afirma que la esperanza de la sociedad en el siglo XXI está depositada en la distribución equitativa del conocimiento, de la ciencia y de la tecnología, como derecho humano, bajo la expectativa de que la concreción de este objetivo contribuirá a movilizar los otros derechos humanos. En este marco, en el que "educar es humanizar", re-significamos y re-enunciamos las capacidades consideradas fundamentales para enfrentar los desafíos de una vida más digna para todos.

En el informe de la UNESCO “Educación de calidad para todos. Un asunto de derechos humanos" (2007) se señaló que la educación no sólo debe promover las competencias básicas tradicionales, sino que también ha de proporcionar los elementos necesarios para ejercer plenamente la ciudadanía; contribuir a una cultura de paz y a la transformación de la sociedad. Por ello, afirmamos que enseñar a aprender a aprender, a convivir y a emprender ${ }^{2}$ se ha vuelto un imperativo si se pretende dar respuesta a los retos propios de la complejidad de la vida cotidiana, social, política -regional, nacional y mundial- y, al mismo tiempo, conciliar esta ineludible responsabilidad social con un proyecto personal de vida (Ferreyra \& Peretti, 2006).

En este contexto, pensar la acción educativa desde el desarrollo de capacidades implica potenciar la función de desarrollo de cultura y de humanidad que es inherente a la educación. Se trata, de pensar un proyecto que imagina, que construye la posibilidad de conciliar el presente y el futuro, lo personal y lo colectivo, los intereses individuales

2 Los objetivos del aprender a emprender tienen que ver con la preparación de ciudadanos íntegros como verdaderos autores de la transformación de su entorno, mediante el abordaje de un conjunto de capacidades relacionadas con la iniciativa personal, la adaptación a los cambios, el desarrollo del pensamiento creativo, el espiritu crítico y el aprender a trabajar en y con la incertidumbre. 
y los sociales; que permita a las personas pensar y actuar de una manera constructiva, ocuparse de su propio crecimiento, vincularse con otros y generar cambios en sus contextos (UNICEF, 2006). Todo esto conduce, inmediatamente, a advertir la necesidad e importancia de un trabajo conjunto (del sistema educativo y de los diferentes sectores sociales) y continuo (durante toda la vida), para suscitar el efecto multiplicador que es propio de los procesos de adquisición y desarrollo de capacidades: una acción transformadora que se manifiesta en los sujetos y que, desde ellos y con ellos, se proyecta a los grupos, las organizaciones, las comunidades, la sociedad toda.

Si el desarrollo de capacidades se orienta a garantizar a todos su formación como personas críticas, creativas, capaces de generar nuevos conocimientos y de disponer de ellos para actuar en diferentes contextos, se constituye en una vía privilegiada para la mejora de la calidad humana de los sujetos, para la democratización de la educación bajo el principio de igualdad de oportunidades, para dar respuesta a los problemas presentes y futuros con visión transformadora. Para ello es necesario que la educación responda a los principios de calidad, pertinencia y relevancia. Un proceso formativo de calidad es aquel que asegura a los sujetos la apropiación de los bienes de la cultura y les permite construir un conjunto de saberes que los habilite para intervenir creativamente en su contexto, producir y aportar. Se trata, entonces, de promover aprendizajes relevantes y significativos desde el punto de vista de las exigencias sociales y de desarrollo personal y que, a la vez, consideren las características y necesidades de cada persona, mediatizadas por el contexto social y cultural en que vive.

\section{Desarkollo de capacidades COMO PROYECTO CURRICULAR $Y$ PEDAGógICO}

El énfasis antes puesto en el conocimiento como contenido escolar/producto y en la enseñanza como transmisión de contenidos, se traslada a la propuesta de adquisición y desarrollo de capacidades que habiliten a los estudiantes para afrontar los desafíos de los nuevos contextos y escenarios. Esto no conduce, sin embargo, a un recorte arbitrario del currículo o a la incorporación de nuevos contenidos a las propuestas curriculares. Se trata de pensar en la adquisición y el desarrollo de aquellas capacidades que permitan abordar los diferentes tipos de contenidos (conceptos/ideas estructurantes, procedimientos y/o actitudes básicas) inherentes a los núcleos de aprendizajes prioritarios $^{3}$, que son imprescindibles tanto para el desempeño escolar de los estudiantes como para la resolución de las múltiples situaciones que se les presentan en la vida cotidiana.

Por su contribución a la construcción de lo común, el currículum expresa la dimensión pública de la escolaridad, pues da cuenta de aquello que se considera valioso para hacer efectiva la igualdad en la distribución de saberes; comunica el tipo de experiencias educativas que se desea y espera ofrecer a los estudiantes -niños, adolescentes, jóvenes y adultos- consideradas fundamentales para su desarrollo y para su participación social

3 En Argentina, y en el marco de la Ley de Educación Nacional, desde el Consejo Federal de Educación, se ha acordado la aprobación de los Núcleos de Aprendizajes Prioritarios (NAP) con la intención de promover la integración del Sistema Educativo Nacional. Los NAP para los diferentes niveles y ciclos fueron elaborados por representantes políticos, técnicos, supervisores y docentes de las provincias argentinas y en Buenos Aires y por los equipos técnicos del Ministerio de Educación Nacional, y aprobados por las autoridades educativas de todas las jurisdicciones en la sesión del Consejo del 13 de octubre de 2004. 
y expresa el compromiso del Estado como articulador de las diferentes demandas de la sociedad, de los estudiantes y de las instituciones que los educan (Terigi, 1999).

En este marco la necesidad de redefinir qué es lo básico o fundamental que han de aprender los estudiantes es una constante en el debate pedagógico. Más allá de las respuestas teóricas que se han dado a esta inquietud, lo cierto es que la sobrecarga de contenidos que caracteriza a los currículos en muchos países atenta contra la calidad de la educación. Ante los nuevos requerimientos de la sociedad y las demandas de los distintos actores sociales,

...se van agregando nuevos contenidos al currículo, sin eliminar otros en la misma medida, y sin reflexionar suficientemente sobre qué aprendizajes debieran ser asumidos por la educación escolar y qué otros deberían ser responsabilidad de otros agentes e instancias educativas (UNESCO, 2007, p. 38).

La decisión sobre qué enseñar debería realizarse considerando cómo se contribuye a alcanzar los fines de la educación, en la búsqueda de un equilibrio entre las demandas sociales, las del desarrollo personal y las derivadas del proyecto político, social y cultural que se desea promover mediante la educación escolar (Coll \& Martín, 2006). Responder a estas diferentes exigencias no es una tarea fácil; por el contrario, implica optar -tras reflexionar- sobre lo imprescindible y lo deseable: una diferenciación que, sin estar libre de dificultades, puede servir de ayuda para seleccionar qué contenidos enseñar y qué capacidades desarrollar, puesto que, de no hacerlo, pueden derivar consecuencias importantes para el futuro aprendizaje. Se hace aquí presente la dimensión del pensar la educación vinculada con aquella acepción que consignábamos en la introducción de este artículo, "Reflexionar, examinar con cuidado algo para formar dictamen".

El gran desafío de un proyecto curricular y pedagógico centrado en la adquisición y desarrollo de capacidades no consiste sólo en poder definir cuáles son las fundamentales y cómo hacer para desarrollarlas, sino también a partir de qué contenidos debieran ser promovidas. En el documento El desarrollo de capacidades en la escuela secundaria. Un marco teórico (Ministerio de Educación, Argentina, 2010), se expresa que "La entrada simultánea por capacidades básicas y por saberes fundamentales pareciera ser central a la hora de definir lo que debe ser enseñado y aprendido" (p. 54).

También es contenido del debate la relación entre capacidades y saberes conceptuales. $\mathrm{Al}$ respecto el documento ya aludido aporta una postura:

Las capacidades no pueden ser desarrolladas en el vacío. La red de contenidos conceptuales disciplinares se debe constituir en el marco de referencia sobre el cual trabajar en relación con el desarrollo de capacidades. Esto no debe interpretarse como algo secuencial en el sentido de disponer primero de la red de contenidos para luego desarrollar capacidades, sino que se tratará de un trabajo interrelacionado. La intención del trabajo por capacidades no implica desentenderse de la enseñanza de contenidos conceptuales, sino más bien revisar la organización, la secuencia y la importancia relativa que le atribuimos a los distintos conceptos disciplinares (Ministerio de Educación, Argentina, 2010, pp. 18-19). 
También debe ser revisada la tendencia a pensar que cierta y determinada capacidad es privativa de ciertas disciplinas o áreas curriculares específicas (por ejemplo, la capacidad de comprensión y producción de textos orales y escritos, como privativa del área de lengua y literatura, o la de resolución de problemas restringida al ámbito de la Matemática). Lo cierto es que el carácter integral e integrador de las capacidades hace que estas atraviesen de manera horizontal y vertical las propuestas curriculares de los distintos niveles y modalidades del sistema educativo y deban y puedan ser abordadas en las distintas disciplinas, áreas o espacios curriculares para el logro de más y mejores aprendizajes para todos.

Avanzar en un desarrollo pedagógico centrado en capacidades implica, una nueva lectura de los marcos y materiales curriculares vigentes desde una perspectiva reflexiva de la práctica docente y desde la lógica del trabajo de gestión institucional. Supone dar la posibilidad a los actores involucrados -tanto docentes como estudiantes- de enfrentar diferentes contextos y situaciones, promoviendo su participación e involucramiento crítico. En consecuencia, contribuye a hacer efectivo el derecho a una educación de calidad.

\section{Capacidades fundamentales: ¿CUÁL ES SU NATURALEZA Y POR QUÉ SON FUNDAMENTALES?}

Consideramos capacidades fundamentales a aquellas que se caracterizan por un alto grado de complejidad; se desarrollan de manera conectiva y procuran el máximo despliegue de las potencialidades de la persona. En este sentido, cada área de conocimiento o disciplina -desde su particularidad, naturaleza y finalidades formativas- contribuye al fortalecimiento, desarrollo y adquisición de las capacidades fundamentales, ya que estos logros se alcanzan al operar con diferentes contenidos, cada uno de los cuales dejan su impronta sobre ese desarrollo, y lo enriquecen.

Entendemos que las capacidades contempladas en este artículo no son las únicas que se pueden abordar en la escuela, pero son las que consideramos fundamentales porque:

- están estrechamente relacionadas con las grandes intencionalidades formativas del currículum de los diferentes niveles del sistema educativo;

- resultan más potentes para la apropiación de conocimientos;

- tienen "incidencia directa, relevante y positiva en los itinerarios escolares de los alumnos" (Ministerio de Educación, Argentina, 2010, p. 55);

- $\quad$ son aquéllas que todos los estudiantes tienen que desarrollar pues se trata de las necesarias para que puedan conocer, comprender, interpretar y participar en el mejoramiento de su calidad de vida $y$, por ende, la de su comunidad de pertenencia, tomando decisiones fundamentadas que les permitan continuar aprendiendo más allá de la escolaridad, dentro de un proceso de educación permanente (Ferreyra \& Peretti, 2008).

Entonces, para poder cumplir con las funciones formativas de los diferentes niveles educativos, la institución escolar trabaja para el desarrollo y adquisición de aquellas capacidades que deberán potenciarse para que todos los estudiantes se apropien de los aprendizajes considerados prioritarios. Si 
bien para su descripción van a ser abordadas por separado, es fundamental tener presente que, en las prácticas, aparecen fuertemente vinculadas.

\section{Comprensión y producción de textos orales y escritos}

Asumir la enseñanza desde un enfoque centrado en el desarrollo de capacidades inherentes a la comprensión y producción oral, la lectura y la escritura, entendidas en tanto acciones lingüístico-comunicativas, mediadas por la sociedad y la cultura y que suponen numerosas estrategias de carácter cognitivo y metacognitivo, de las cuales los estudiantes deben apropiarse, implica considerarlas como aprendizaje prioritario y eje central de la enseñanza. Esta capacidad compleja supone el abordaje de las actividades lingüísticas claves, hablar, escuchar, leer, escribir, en el marco de las diferentes prácticas sociales de lenguaje que les dan sentido. Por su carácter genérico y transversal a todos los aprendizajes, su desarrollo es incumbencia de todas las áreas y disciplinas curriculares.

Desde un punto de vista cognitivo, comprender es una acción compleja que se realiza por medio de distintas estrategias de diferente índole, encaminadas a lograr una representación semántica del contenido del texto y de sus implicancias -lo que el texto dice y le dice al lector- y pragmática: por qué y para qué lo dice.

Ser capaz de producir textos orales o escritos supone, ante todo, estar en condiciones de construir un mensaje que permita dar respuesta a una necesidad de comunicar sentido y establecer una interacción sociocomunicativa. Esta producción requiere re- presentarse la escritura como un problema por resolver ya que escribir demanda poner en juego una serie de saberes: conocimientos lingüísticos (gramática oracional y textual, aspectos normativos, etc.), conocimiento del tema, conocimientos sobre el proceso de composición (planificar, redactar, revisar, corregir) y conocimiento de las estrategias para resolver las demandas de la situación comunicativa.

La capacidad de comprender y producir textos orales y escritos se vincula plenamente con los objetivos de la política educativa por cuanto garantiza a todos los estudiantes el acceso a las condiciones para "tomar la palabra", es decir, poder expresar lo que se siente, se sabe y se necesita, en forma oral o por escrito, lo cual allana el camino a la inclusión y la igualdad.

\section{Abordaje y resolución de situaciones problemáticas}

Un problema puede definirse como toda situación nueva o sorprendente que un individuo o grupo desea modificar y de la cual se conoce el punto de partida y a dónde se desea llegar, pero se desconoce un procedimiento directo para lograrlo (Pozo, et ál., 1995). Por ello, la descripción de una situación no retrata un problema hasta que no se incluye en el análisis a la persona que intentará modificar dicha situación; es decir, existen situaciones que no serían consideradas un problema si quien las resuelve ya sabe cómo hacerlo ${ }^{4}$.

4 Con el tiempo, sea por entrenamiento específico o por el propio desarrollo normal (evolución cognitiva e interacciones sociales), adquirimos progresivamente procedimientos de solución a diferentes tipos de situaciones que van dejando de ser problemas. A lo largo de nuestra vida aprendemos modos de eliminar problemas culturalmente identificados, desde el dominio de la gramática hasta los juicios sobre la validez de un silogismo. 
Existen al menos dos modos distintos de resolver los problemas a los que nos enfrentamos, uno, la transmisión cultural directa de la solución de dichos problemas de manos de alguien que ya no la considera un problema, con indicaciones precisas de cómo se resuelve y con alguna clase de entrenamiento que permita automatizar su solución; el otro modo consiste en dejar que sea el propio individuo quien explore el problema y vaya construyendo soluciones adecuadas, con o sin ayuda y supervisión de expertos.

Si lo que preocupa no es tanto enseñar una respuesta de la manera más rápida posible, sino utilizar cada problema que se va presentando como una nueva oportunidad para la adquisición y desarrollo de las capacidades de la persona. Entonces, será necesario dar tiempo al estudiante para que se involucre en el problema e intente encontrar modos de abordarlo por sus propios medios. Esto supone formarse una idea integral de la situación a la que se refiere el problema, identificar los aspectos que lo componen y los sujetos implicados; buscar varios caminos para solucionarlo (obtener datos, verificarlos, dar respuesta a lo que se ignora), seleccionar uno, aplicar el camino elegido, recorrerlo para solucionar el problema. Esto implica ser capaz de codificar, almacenar, recuperar y transformar la información, además de monitorear y evaluar la propia actuación.

En el ámbito de la propuesta didáctica, resulta imprescindible poder diferenciar entre problemas y ejercicios. Mientras estos tienen por finalidad el desarrollo de destrezas que se aplicarán luego a situaciones idénticas o muy similares a las que se utilizaron durante el entrenamiento, los problemas buscan el desarrollo de una habilidad com- pleja cuyo campo de acción se pretende que sea tan amplio como sea posible. Así, si en la escuela se pretende tomar un rol activo en el desarrollo de la capacidad de abordaje y resolución de problemas, deberán darse a los estudiantes oportunidades frecuentes y variadas de enfrentarse a situaciones desafiantes, apoyadas en los contenidos de las distintas áreas curriculares. Pero para ello es necesario percibir las implicaciones de las diferentes aproximaciones que realizan los estudiantes; si son fructíferas o no y qué podrían hacer en lugar de eso; decidir cuándo intervenir, qué sugerencias ayudarán sin restar autonomía. Finalmente, corresponde que el docente supere la propia ansiedad por tener que trabajar sin saber todas las respuestas posibles.

\section{Comprensión y explicación de la realidad social y natural, empleando conceptos, teorías y modelos}

La comprensión del mundo que nos rodea en toda su complejidad es una capacidad humana que se desarrolla a través de diversas intervenciones culturales, entre las cuales la enseñanza escolar ocupa un papel central debido a su carácter sistemático, gradual y planificado.

Las construcciones mentales que utilizamos para comprender el mundo y materializar esa comprensión en mensajes comunicables son los conceptos, los modelos y las teorías. Desde la tarea docente, desarrollar estas construcciones cognitivas y ayudar a los estudiantes a que puedan usarlas para "leer los mundos" supone intervenciones tales como proporcionar claves disciplinares para establecer un recorte adecuado de un 
fenómeno o situación; ayudar a reconocer lo que permanece y lo que cambia; acompañar en la identificación de los hechos que desencadenan un suceso o fenómeno, o que son consecuencias de éste; enseñar a tener en cuenta los múltiples factores que operan en una situación (multicausalidad); enseñar a tener en cuenta las diversas interpretaciones de hechos y acontecimientos a través de la historia y en diferentes culturas; ayudar a reconocer los condicionantes geográficos, históricos y éticos de los avances científicos y tecnológicos, entre otras.

Todo esto implica que la capacidad pueda enriquecerse como resultado de un proceso consciente de aprendizaje impulsado por la acción educativa, situándose en el punto de vista de un contexto determinado de producción de conocimiento (por ejemplo la historia, la biología, la matemática, la tecnología, etc.) y empleando las preguntas relevantes, los modelos explicativos, las formas de pensar y de hacer propias de estos contextos, como herramientas para abordar el estudio de una situación, objeto, fenómeno o proceso. Pero esta capacidad también puede ser necesaria al plantearse preguntas sobre una situación, objeto, fenómeno o proceso complejo para el que no sea suficiente la lectura particular de una disciplina científica, por lo que en estos casos se requieren intervenciones planificadas a nivel de la escuela que signifiquen oportunidades para que surjan estas preguntas multi o interdisciplinares y que luego puedan derivarse a tratamientos disciplinares convergentes.

Utilizar de manera significativa y relevante los conocimientos adquiridos para analizar más profundamente una situación, objeto, fenómeno o proceso del contexto natural y social, permitiría operar sobre la realidad con mayor conocimiento y compromiso.

\section{Trabajo en colaboración}

para aprender a relacionarse

\section{e interactuar}

El valor de la educación reside, hoy más que nunca, en su potencial para contribuir a la construcción de un mundo para todos. Esto sólo será posible en la medida en que la escuela ofrezca a los estudiantes experiencias en las que el aprender a ser, a saber y a hacer se plantee como una empresa colectiva, basada en el saber convivir, en un simultáneo reconocimiento de lo que en tanto sujetos humanos los iguala y de todo lo que los hace diferentes.

Esta capacidad se refiere a la posibilidad de interacción y supone el desarrollo y adquisición de habilidades para receptar las ideas de los demás (escucha activa) y exponer las propias; contribuir a lograr los objetivos del trabajo conjunto; mejorar las prácticas cooperativas; apreciar los frutos de esas prácticas; cooperar con el desarrollo del entorno y valorar la tarea grupal.

Receptar las ideas de los demás y exponer las propias implica aprender con otros y de otros. Tanto la expresión como la escucha atenta y solidaria son requisitos básicos para el desarrollo de esta capacidad.

Elaborar acuerdos, establecer conclusiones, solucionar conflictos y sostener consensos son algunas de las habilidades que se pueden desarrollar a partir del trabajo con otros. La escuela y el aula constituyen uno de los primeros ámbitos en donde se aprende (o no) a valorar el trabajo conjunto y estas experiencias tendrán su impronta en 
la futura vida laboral y/o profesional de los estudiantes.

Los problemas son resueltos por el equipo, $\mathrm{y}$ quienes intervienen en él desarrollan las habilidades de liderazgo, comunicación, confianza, toma de decisiones, solución de conflictos y responsabilidad por los resultados.

La capacidad de trabajo en colaboración para aprender a relacionarse e interactuar supone reconocer la alteridad y aceptar al otro en tanto otro diferente. De esta manera, colabora con el proceso de aprendizaje en el que nos reconocemos como ciudadanos en un mundo complejo, culturalmente diverso y desigual. La escuela debe propiciar que los estudiantes superen el egocentrismo y potencien su capacidad para distinguir entre el propio punto de vista y la perspectiva de los demás.

\section{Pensamiento crítico y creativo}

El contexto actual -caracterizado por profundos procesos de transformación; en el que impera lo audiovisual; donde los medios de comunicación tienen una presencia $\mathrm{y}$ un poder enormes, que pueden reducir el ser ciudadano a un mero ser espectador y donde todo queda al mismo nivel de significación- reviste una gran importancia el fortalecimiento de la capacidad del ciudadano para conmoverse, para discriminar, para pensar y actuar. Se trata de formar ciudadanos críticos y creativos, capaces de dirigir su visión, de observar, de situarse en el mundo, de desnaturalizarlo, de interpretarlo y comprenderlo y, así, poner en práctica nuevas ideas originales e innovadoras que contribuyan a crear un nuevo orden social.

El pensamiento contiene aspectos críticos y creativos. Por lo tanto, es importante que cualquier tentativa de mejorar las capacidades del pensamiento preste atención a ambas dimensiones. El pensamiento crítico es necesario para analizar discusiones y para la toma racional de decisiones, mientras que el pensamiento creativo es necesario para desarrollar alternativas deseables. El pensamiento creativo implica la generación de ideas, el pensamiento crítico se refiere a su evaluación. Ambos se correlacionan.

Pensar críticamente es buscar argumentos para asumir o refutar lo que se sabe o piensa. El pensamiento crítico cuestiona las creencias infundadas, los prejuicios, y trata de sustituirlos por ideas argumentalmente sostenidas. De la misma manera, examina los supuestos conocimientos, rescata lo que tengan de verdad y tantea nuevas verdades, reconociendo que ellas son construcciones humanas susceptibles de nuevas críticas y refutaciones.

En síntesis, el pensamiento creativo complementa al pensamiento crítico, le da libertad para generar nuevas ideas y arribar a una solución original e innovadora. En este sentido, aprender a pensar de manera crítica y creativa es aprender a ser sujeto; es aprender a construir la propia subjetividad; es valorarse como ser capaz de producir significados e interpretar el mundo; es atreverse a pensar, a hacer y a ser.

El breve desarrollo de cada capacidad que hemos presentado nos ha permitido mostrar el estrecho lazo que existe entre el desarrollo de cada una de las capacidades fundamentales y la adquisición o despliegue de las otras. Más aún, es posible afirmar que el desarrollo y adquisición de una capacidad se potencia cuando se pone en el marco del trabajo con otras. 


\section{En cada escuela y en cada aula}

Asumir con responsabilidad el desarrollo de un currículum centrado en el trabajo con capacidades supone un cambio en la cultura pedagógica de la institución escolar y de los docentes. Es preciso el trabajo colaborativo para operar sinérgicamente, ya que en el desarrollo y adquisición de las capacidades fundamentales están comprometidas todas las áreas desde la especificidad de contenidos que les es propia y, simultáneamente, con la mirada puesta en la interrelación de los conocimientos. En consecuencia, focalizar la enseñanza en el desarrollo y fortalecimiento de las capacidades fundamentales implica aprendizajes cuya consecución no es responsabilidad exclusiva del accionar de un área, asignatura o disciplina determinada, sino que requiere de estrategias de enseñanza convergentes y sostenidas a lo largo del tiempo, que generen nexos y conexiones entre distintos campos del saber y del hacer.

Cabe aclarar que no es suficiente la mención de las capacidades en las planificaciones generales, sino que ellas deben estar presentes en la preparación de cada instancia de aprendizaje y en la previsión de los modos en que se irán evaluando (valorando) los logros que los estudiantes van alcanzando.

Las principales técnicas didácticas que se sugieren para el desarrollo y adquisición de las capacidades, así como para la adquisición de conocimientos y la asunción de actitudes y valores son el aprendizaje basado en problemas, el aprendizaje orientado a proyectos, el estudio de casos y el aprendizaje cooperativo. Las estrategias de enseñanza surgirán de las adaptaciones y contextualizaciones que el docente realice de estas técnicas. Por otra parte, se procurará que las actividades permitan al estudiante desempeñar un papel activo, dándole la oportunidad de planificar, de participar en el desarrollo de las tareas y de sus resultados, y de revisar y mejorar sus esfuerzos.

Es necesario que el estudiante comprenda la importancia del desarrollo y adquisición de sus capacidades para su crecimiento integral y desempeño exitoso en el mundo actual. Por ello, el docente debe reconocer las habilidades y estrategias implícitas en la capacidad que pretende desarrollar, valorar el nivel alcanzado al respecto por los estudiantes, comunicar el sentido y la importancia de su adquisición y explicar en qué consiste.

Orientar la acción de enseñanza hacia el desarrollo y adquisición de capacidades, supone convertir a la escuela y al aula en verdaderas comunidades de aprendizaje, cuyas características distintivas inciden decisivamente en los procesos, modalidades y criterios de evaluación, si es que estos pretenden ser coherentes con el enfoque en cuestión. Para ello, será importante, entonces, tener en cuenta:

- Que si una de las condiciones básicas del desarrollo y adquisición de capacidades es que se ofrezca al estudiante la posibilidad de participación en variadas y asiduas ocasiones de aprendizaje auténticas y situadas, la evaluación ha de adscribir también a esta condición, configurándose a través de tareas que no descontextualicen los conocimientos, brinden tiempo y posibilidad de exploraciones, admitan múltiples vías de solución, y no determinen explícitamente las acciones adecuadas. 
- Que el aprendizaje no es una empresa individual y solitaria, sino una tarea compartida en la que se involucran, cooperativamente estudiantes, docentes y también las familias y otros adultos de la comunidad. Una tarea en la cual inciden, además, el entorno y los recursos e instrumentos que están a disposición. Ninguna evaluación será auténtica en la medida que no tomen en cuenta estos factores.

- $\quad$ En la búsqueda y construcción de conocimientos importa no sólo la capacidad personal que se pone en juego, sino también los valores compartidos y el espíritu cooperativo.

- El sentido de la evaluación ha de fundarse en el seguimiento de los logros progresivos de los estudiantes y no en la mera comprobación (o sanción) de sus obstáculos, dificultades y/o fracasos. Sólo así será posible asumir el proceso de enseñanza y aprendizaje como instancia para el desarrollo de las potencialidades de los sujetos.

- La evaluación ha de permitir a los estudiantes la toma de conciencia acerca de sus dificultades y sus logros y, al mismo tiempo, alentarlos a asumir la responsabilidad de sus posibilidades de avance y mejora. Para ello es necesario que los procesos de evaluación, más allá de su valor instrumental en función de la acreditación, sean concebidos de manera que puedan constituir, en sí mismos, nuevas y valiosas ocasiones de aprendizaje, que promuevan en los estudiantes deseos de superación y en los docentes, la revisión y la correspondiente reformulación y/o consolidación de las prácticas.
- $\quad$ En tanto el aprendizaje es siempre contextualizado y diferente según el individuo, no cabe pensar la evaluación desde una perspectiva homogeneizadora.

- Dada la importancia de la actividad metacognitiva en el efectivo desarrollo y adquisición de capacidades, la evaluación también ha de orientarse a valorar la progresiva toma de conciencia del estudiante acerca de sus "modos" de afrontar y resolver situaciones nuevas; los avances en las estrategias de monitoreo, autorregulación y autocorrección; la adquisición de autonomía...

- Es fundamental que en una situación de evaluación exista en los estudiantes una conciencia explícita de los objetivos que la orientan y puedan participar de la determinación de lo que se espera que ellos estén en condiciones de saber y poder hacer. Esta condición contribuye a que se satisfaga la necesidad de que el estudiante otorgue sentido a la tarea y, consecuentemente, se involucre en ella como participante y no como objeto.

\section{REFLEXIONES FINALES}

Pensar la educación es, primordialmente, pensar el futuro y en el futuro. ¿Y cuándo y cómo pensamos la educación como una manera de proyectar y edificar futuro?

Cuando reflexionamos y examinamos las condiciones del presente de cada uno de nuestros estudiantes y, al mismo tiempo, los contextos complejos, cambiantes, exigentes que ellos habitan y las circunstancias (muchas veces difíciles y hasta adversas) en las que se desarrollan sus vidas. Entonces, generamos, como sociedad y como colectivo docente, situaciones que los convierten 
en protagonistas de experiencias educativas que les aseguren aprendizajes vinculados no sólo con conocimientos puntuales de corta duración, sino aprendizajes significativos, funcionales, innovadores, que les permitan transformar y transformarse.

Cuando en cada niño, joven o adulto que se está educando vemos no la carencia o el déficit, lo que les falta, sino la potencialidad y lo que pueden lograr en el proceso de de-

\section{REFERENCIAS}

Carriego, C. (2005). Los desafíos de la gestión escolar. Una investigación cualitativa. Buenos Aires: La Crujía.

Casas, L., et ál. (2007). Guía para el desarrollo de capacidades. Lima: Ministerio de Educación de la República del Perú.

Coll, C. (2006). Lo básico en la educación básica. Reflexiones en torno a la revisión y actualización del currículum de la educación básica. Transatlántica de Educación, 1(1). México: Editorial Santillana.

Coll, C. \& Martín, E. (2006). La vigencia del debate curricular. En: El currículo a debate. PREALC, 3. Santiago de Chile: OREALC/UNESCO.

Delors, J. (1996). Informe: La educación encierra un tesoro. Madrid: Editorial Santillana - UNESCO.

Ferreyra, H., et ál. (2005). Claves de buena calidad para la educación básica Argentina. Diagnósticos, desafíos y propuestas. Córdoba, Argentina: Editorial Mimeo.

Ferreyra, H. \& Peretti, G. (comp.) (2006). Diseño y gestión de una educación auténtica. Buenos Aires: Novedades Educativas.

Ferreyra, H., Peretti, G., et ál. (2008). De aprendizajes, competencias y capaci- sarrollo de sus capacidades, si atendemos, al mismo tiempo, a su individualidad y a su condición de sujetos sociales.

Cuando imaginamos y diseñamos, desde el presente, proyectos políticos, curriculares y pedagógicos orientados a la construcción de escenarios futuros en donde lo que hoy deseamos es que adquiera la dimensión de lo posible.

dades en la educación primaria. Desandando caminos para construir nuevos senderos. Revista Iberoamericana de Educación, 47(3). OEI. Recuperado el 2 de junio de 2009 desde [http://www.rieoei. org/2702.htm].

Ferreyra, H. \& Orrego S. (coord.); Peretti, G., et ál. (2007). Competencias prioritarias en la educación primaria. Paraná, Argentina: Editorial Mimeo.

Gobierno de Córdoba \& Ministerio de Educación (2002). Las competencias educativas prioritarias. Un compromiso con la calidad. Cuadernos para pensar, hacer y vivir la escuela. (2). Córdoba, Argentina: Autor.

Ministerio de Educación (Argentina) (2010). El desarrollo de capacidades en la escuela secundaria. Un marco teórico. Buenos Aires: Ministerio de Educación - UNICEF - OEI. Asociación Civil Educación para Todos. Recuperado el 20 de marzo de 2011 desde [http://www.unicef.org/argentina/spanish/Cuaderno_1.pdf].

Ministerio de Educación, Ciencia y Tecnología de la Nación (Argentina). Secretaría de Educación. Subsecretaría de Equidad y Calidad. Dirección Nacional 
de Gestión Curricular y Formación Docente (2001). Desarrollo de capacidades en EGB 3 y educación polimodal. Buenos Aires: Autor.

Montero, A. (2008). Competencias educativas y objetivos como capacidades. Escuela, No. 3.783. Universidad de Sevilla.

ONU/ ECOSOC/ UNESCO (2003). Right to Education. Scope and Implementation. General Comment 13 on the Right to Education. Article 13 of the International Covenant on Economic, Social and Cultural Rights. París: UNESCO. Recuperado el 3 de abril de 2011 desde [http://unesdoc.unesco. org/images/0013/001331/133113e.pdf].

Pozo, J. I., Postigo, Y. \& Gómez Crespo, M. A. (1995). Aprendizaje de estrategias para la solución de problemas en ciencias. Alambique - Didáctica de las Ciencias Experimentales, (5), Barcelona, España.

Terigi, F. (1999). Currículum. Itinerarios para aprender un territorio. Buenos Aires: Editorial Santillana.

UNESCO (2000). Documentos de referencia para la educación para todos. Recuperados el 20 de marzo de 2011 desde [http:// www.unesco.org/new/es/education/ themes/leading-the-international-agenda/efareport/background-papers/].

UNESCO (2007). Educación de calidad para todos. Un asunto de derechos humanos. Documento de discusión sobre políticas educativas en el marco de la II Reunión Intergubernamental del Proyecto Regional de Educación para América Latina y El Caribe. Oficina Regional de Educación para América Latina y El Caribe (OREALC/UNESCO). Buenos Aires. Recuperado el 20 de marzo de 2011 desde [http://unesdoc.unesco.org/images/0015 /001502/150272s.pdf].

UNESCO/OREALC (2002). Proyecto regional de educación para América Latina y El Caribe (PRELAC). Santiago de Chile: UNESCO. Recuperado el 3 de junio de 2009 desde [http://unesdoc.unesco. org/images/0015/001516/151698s. pdf\#151582].

UNICEF (2006). Desarrollo de capacidades para el ejercicio de la ciudadanía. Buenos Aires: Autor. 